\title{
NEWLY-DETECTED GLUCOSE DISTURBANCES IN PATIENTS UNDERGOING CORONARY ANGIOGRAPHY FOR KNOWN OR SUSPECTED CORONARY ARTERY DISEASE
}

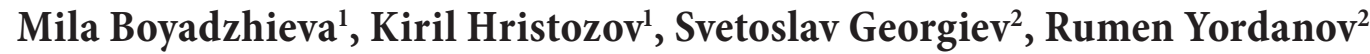 \\ ${ }^{1}$ Clinic of Endocrinology and ${ }^{2}$ Second Clinic of Interventional Cardiology, \\ St. Marina University Hospital of Varna
}

\begin{abstract}
PURPOSES: Glucose disturbances are common in patients with coronary artery disease (CAD), however, usually, they remain undiagnosed. The aim of this study was to estimate the newly-diagnosed glucose abnormalities in patients undergoing coronary angiography for known or suspected CAD.

MATERIAL AND METHODS: A routine oral glucose tolerance test (OGTT) was applied in 96 consecutive patients without previous history of type 2 diabetes mellitus (T2DM) undergoing coronary angiography. Glucose tolerance was defined according to WHO-2006 criteria by OGTT performed within a week after hospital discharge.

RESULTS: Glucose disturbances prevailed over normoglycemia as $64,58 \%$ of the patients demonstrated hyperglycemia while $35,42 \%$ presented with normal glucose tolerance (NGT). Overall, 120 min-hyperglycemia $(\geq 7,8 \mathrm{mmol} / \mathrm{L})$ was found out in $52,08 \%$ of the participants and only $12,5 \%$ of the cases had isolated fasting hyperglycemia (fasting plasma glucose, $\mathrm{FPG} \geq 6,1 \mathrm{mmol} / \mathrm{L}$ and postchallenge glucose $<7,8 \mathrm{mmol} / \mathrm{L}$ ). Based on plasma glucose values such as FPG and 2-hour post-OGTT glucose, the proportion of patients with newlydiagnosed T2DM, impaired glucose tolerance (IGT) and impaired fasting glucose (IFG) was $26,04 \%, 28,12 \%$ and $10,42 \%$, respectively. Some $20 \%$ of the newly-diagnosed T2DM patients reached a diagnostic FPG value only, 32\% reached 120 min.-plasma glucose (PG) value only while $48 \%$ met combined criteria.

CONCLUSION: Glucose abnormalities identified by OGTT are more common than normoglycemia - in $64,58 \%$ versus $35,42 \%$ of the patients undergoing coronary angiography for known or suspected CAD. This finding strongly suggests that OGTT is the most valuable tool for the early detection of disturbed glucose regulation and should be performed routinely in the patients with known or suspected CAD.
\end{abstract}

Key words: type 2 diabetes mellitus, coronary artery disease, oral glucose tolerance test, impaired fasting glucose, impaired glucose tolerance

\section{INTRODUCTION}

Glucose abnormalities are widespread among patients with coronary artery disease (CAD) and have serious prognostic implications. In fact,

Address for correspondence:

Mila Boyadzhieva, MD

Clinic of Endocrinology

St. Marina University Hospital of Varna

1 Hristo Smirnenski Str., 9010 Varna, Bulgaria

e-mail:milaboja@yahoo.com

Received: June 25, 2013

Accepted: July 25, 2013 disturbed glucose metabolism is more prevalent than normoglycemia in CAD patients without known diabetes mellitus and exceeds $60 \%(1,7,8,10,12,16)$. Importantly, in the general population, oral glucose tolerance test (OGTT) doubles the number of patients diagnosed with diabetes mellitus towards fasting plasma glucose (FPG) (3,5\% versus 7,3\%) (3) whereas 
in the coronary population there may be a fivefold increased difference (5,3\% versus $26,9 \%)$ (7). All the stages of abnormalglucose metabolism, even modestly elevated but not within diabetic range glucose levels, carry an increased risk of cardiovascular morbidity and mortality $(4,14)$. Impaired fasting glycose (IFG) and impaired glucose tolerance (IGT) are forms of prediabetes characterized by fasting and postprandial hyperglycemia, respectively. It is noteworthy that the patients with glucose abnormality may have normal fasting but elevated postchallenge glycemia during OGTT, and vice versa.

Two-thirds of the patients with CAD and positive OGTT would remain undetected if only FPG is measured (2). The severity of postchallenge hyperglycemia closely correlates with future cardiovascular $(\mathrm{CV})$ events and total mortality rate (13). Moreover, a 2-hour postchallenge glycemia during OGTT is not only a better predictor of the dysglycemic state than FPG alone but also a better risk predictor for subsequent cardiovascular complications $(5,11)$. The Funagata Study also reveals a higher CV mortality rate in subjects with IGT than in those with IFG (15). In three other studies, CV mortality rate in individuals with IGT is close to that of those with overt type 2 diabetes mellitus (T2DM) and much greater than in the subjects with IFG and normal glucose tolerance (NGT) $(5,6,9)$. Therefore, FPG measurement alone is insufficient for a correct recognition of a substantial proportion of individuals with abnormal glucose regulation among CAD patients. That is why OGTT is recommended for the appropriate classification of glucose abnormalities in patients at high risk, which includes all the individuals with established CV disease.

The aim of our study was to estimate the prevalence of newly-diagnosed glucose abnormalities by OGTT among the patients undergoing coronary angiography for known or suspected $\mathrm{CAD}$ and without known glucose abnormalities.

\section{MATERIAL AND METHODS}

Our study covered a total of 96 consecutive patients without previous history of T2DM undergoing coronary angiography at acute or elective admission for known or suspected CAD. They were 78 males $(81,25 \%)$ and 18 females $(18,75)$ at a mean age of 58,2 $\pm 8,6$ years. Anthropometric measurements included height, weight, waist circumference and calculation of body mass index (BMI). A routine OGTT was performed within a week after hospital discharge in all of them. Glucose tolerance was defined according to WHO-2006 criteria. The patients were free of current infections and corticosteroid therapy. Data concerning risk factors, medical history and concomitant medications were collected. Venous plasma was centrifuged immediately after blood collection and glucose concentrations were determined by hexokinase method at $0 \mathrm{~min}$ (FPG) and $120 \mathrm{~min}$ after glucose load (postchallenge glycemia). Glycated hemoglobin (HbAlc) was measured by immunoassay for the quantitative determination of percentage hemoglobin Alc in whole blood samples on the AxSYM System, Abbott, USA. Coronary angiographies were evaluated by interventional cardiologists, who were not familiar with patients' exact glucometabolic status.

Statistical analysis was done using GraphPad Prism version 5. Data are expressed as means \pm SD or $\mathrm{n}(\%)$. Value of $\mathrm{p}<0,05$ was considered statistically significant. The study was approved by the Ethical Commission of the Medical University of Varna.

\section{RESULTS}

Patients' mean BMI was $30,1 \pm 4,2 \mathrm{~kg} / \mathrm{m}^{2}$ and waist circumference was $105,6 \pm 10,5 \mathrm{~cm}$.

Drug therapy administered at hospital discharge

Table 1. Drug therapy at hospital discharge $(n=96)$

\begin{tabular}{l|c|c} 
Drug types & $\mathrm{n}$ & $\%$ \\
\hline $\begin{array}{l}\text { beta-blockers } \\
\text { statins }\end{array}$ & 83 & 86,45 \\
$\begin{array}{l}\text { angiotensin-converting-enzyme } \\
\begin{array}{l}\text { inhibitors/ angiotensin receptor } \\
\text { blockers }\end{array}\end{array}$ & 81 & 84,37 \\
\hline
\end{tabular}

was summarized on Table 1.

Our results showed that glucose disturbances prevailed over normoglycemia as $64,58 \%$ of the patients demonstrated hyperglycemia while $35,42 \%$ presented with NGT. Overall, 120 min-hyperglycemia $(\geq 7,8 \mathrm{mmol} / \mathrm{L})$ was found out in $52,08 \%$ of the participants and only $12,5 \%$ of the cases had isolated 
Mila Boyadzhieva, Kiril Hristozov, Svetoslav Georgiev et al.

fasting hyperglycemia (FPG $\geq 6,1 \mathrm{mmol} / \mathrm{L}$ and postchallenge glucose $<7,8 \mathrm{mmol} / \mathrm{L}$ ). Based on plasma glucose values such as FPG and 2-hour post-OGTT glucose, the proportion of patients with newlydiagnosed T2DM, IGT and IFG was 26,04\%, 28,12\% and $10,42 \%$, respectively.

Without the postchallenge data provided by OGTT, the corresponding prevalence of abnormal glucose metabolism was much lower (in $40,63 \%$ of the cases) (Fig. 1).

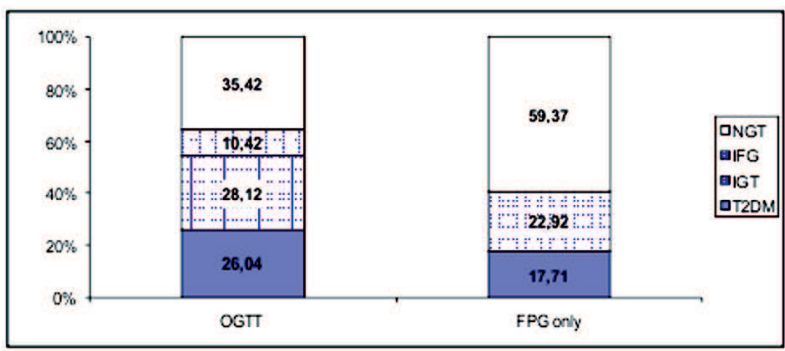

Fig. 1. Comparison of glucose tolerance defined by OGTT (FPG and 120'-postchallenge glucose) or FPG only

Among the subjects with prediabetes, $72,97 \%$ had IGT and $27,03 \%$ had IFG. Moreover, $48,15 \%$ of IGT patients were with normal fasting glycemia $(<5,6$ $\mathrm{mmol} / \mathrm{L}$ ) and $54,05 \%$ of all the patients who met the fasting criterion of 5,6-6,9 $\mathrm{mmol} / \mathrm{L}$ were with IGT or diabetes. Mean FPG of IGT individuals was slightly but significantly higher compared to NGT ones $(5,59 \pm 0,1$ versus $5,26 \pm 0,08 ; p=0,01)$. However, subjects with isolated IGT (FPG $<6.1 \mathrm{mmol} / \mathrm{L}$ ) and NGT showed a similar FPG $(5,40 \pm 0,08$ versus $5,26 \pm 0,08 ; p=0,3$ ) (Fig. 2).

$$
p=0,01 \quad p=0,3
$$

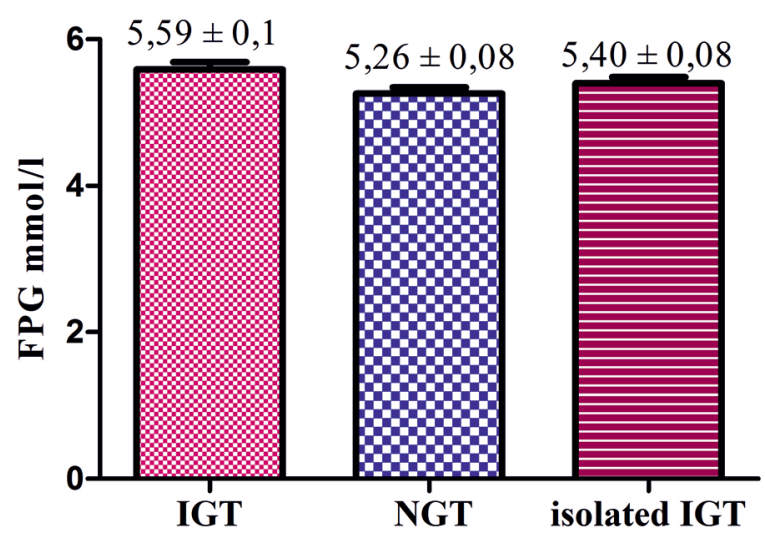

Fig. 2. Comparison of FPG levels between patients with $I G T$, isolated IGT (FPG $<6,1 \mathrm{mmol} / \mathrm{L})$ and NGT
Some $20 \%$ of the newly-diagnosed T2DM patients reached a diagnostic FPG value only, $32 \%$ reached 120 min.-plasma glucose (PG) value only while $48 \%$ met combined criteria. Moreover, $32 \%$ of them had HbAlc $<6,5 \%$. Mean HbAlc was significantly lower in the patients with prediabetes than in the patients with newly-detected T2DM $(5,65 \pm 0,07 \%$ versus $7,14 \pm 0,50 \% ; \mathrm{p}=0,001)$ and similar to that in the subjects with NGT $(5,65 \pm 0,07 \%$ versus $5,46 \pm 0,09 \% ; p=0,1)$. In the patients with HbAlc $<6,0 \%$, glycated hemoglobin correlated only with $120^{\prime}$-glycemia $(\operatorname{Pr}=0,44 ; \mathrm{p}<0,006)$, while in those with $\mathrm{HbAlc} \geq 6,0 \%$, there was a correlation with both FPG and 120 '-postchallenge glucose $(\mathrm{Pr}=0,89$; $\mathrm{p}<0,0001 ; \operatorname{Pr}=0,69 ; \mathrm{p}<0,0007$, respectively).

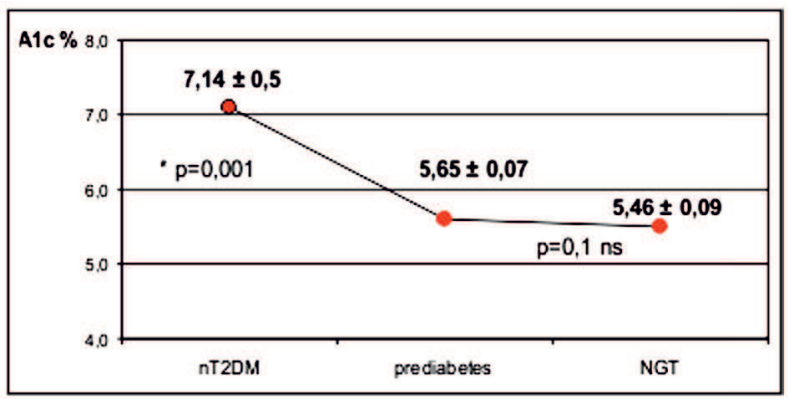

Fig. 3. Percentage of HbAlc in patients with newly-detected T2DM (nT2DM), prediabetes mellitus and NGT

\section{DISCUSSION}

The main findings of our study showed that the newly-detected glucose abnormalities in CAD patients were more common than normoglycemia. Our results are in accordance with previously published data about the high incidence rate of undiagnosed abnormal glucose tolerance discovered by OGTT in angiographied coronary patients independently of the size number $(1,7,16)$. In contrast, glucose abnormalities were markedly more seldom when diagnosis was based on FPG measurement only (7). In our contingent, the 2 -hour post-OGTT hyperglycemia was present in $52,08 \%$ of the cases. Therefore, FPG estimation alone would misdiagnose a substantial proportion of patients with abnormal glucose regulation, including diabetes mellitus and IGT. Although OGTT is recommended for the clear classification of glucose tolerance in these patients 
(2), it has not become a regularly used test in CAD population yet. Known or newly-detected T2DM is a well-known CV risk factor. Several several studies show, however, that IGT and even IFG are associated with an increased risk of CV morbidity and mortality, too $(4,14)$. The observed positive correlation between $\mathrm{HbAlc}<6,0 \%$ and postchallange glycemia and substantial proportion of T2DM and IGT patients who will remain undiagnosed require a routine OGTT.

We performed OGTT soon after hospital discharge without confirmation later on. However, data available demonstrated that determination of the glycemic status in the patients with acute myocardial infarction during hospital stay is reliable and reproducible three months later on (10). This strongly suggests that OGTT is the most valuable tool for the early detection of disturbed glucose regulation and should routinely be performed in the patients with known or suspected CAD.

\section{CONCLUSION}

Glucose abnormalities identified by OGTT are more common than normoglycemia - in $64,58 \%$ versus $35,42 \%$ of the patients undergoing coronary angiography for known or suspected CAD. In addition, more than $50 \%$ of subjects present with postchallenge hyperglycemia. If the classification of glucose abnormalities is done without postchallenge data during OGTT, a substantial proportion of prediabetic and diabetic disturbances would have been not only underestimated but also misclassified. This suggests that OGTT should be performed in any patients with known or suspected CAD.

\section{ACKNOWLEDGEMENTS}

This study was supported by a Grant of the Medical University "Prof. Paraskev Stoyanov" of Varna.

\section{REFERENCES}

1. Bartnik, M., Rydén, R. Ferrari, K. Malmberg, K. Pyorala, M. Simoons, et al. The prevalence of abnormal glucose regulation in patients with coronary artery disease across Europe: the Euro Heart Survey on diabetes and the heart.- Eur. Heart J., 25, 2004, No 21, 1880-1890.
2. Bartnik, M., L. Rydén, K. Malmberg, J. Öhrvik, K. Pyörälä, E. Standl, et al. Oral glucose tolerance test is needed for appropriate classification of glucose regulation in patients with coronary artery disease: a report from the Euro Heart Survey on Diabetes and the Heart.- Heart, 93, 2007, No 1, 72-77.

3. Botas, P., E. Delgado, G. Castano, D. G. Díaz, J. Prieto, F. J. Díaz-Cadorniga. Comparison of the diagnostic criteria for diabetes mellitus, WHO1985, ADA-1997 and WHO-1999 in the adult population of Asturias (Spain).- Diabet. Med., 20, 2003, No 11, 904-908.

4. Coutinho, M., H. C. Gerstein, Y. Wang, S. Yusuf. The relationship between glucose and incident cardiovascular events: a metaregression analysis of published data from 20 studies of 95,783 individuals followed for 12.4 years.- Diabetes Care, 22, 1999, No 2, 233-240.

5. DECODE Study Group, the European Diabetes Epidemiology Group. Glucose tolerance and cardiovascular mortality: comparison of fasting and 2-hour diagnostic criteria.- Arch. Intern. Med., 161, 2001, No 3, 397-405.

6. de Vegt, F., J. M. Dekker, H. G. Ruhe, C. D. Stehouwer, G. Nijpels, L. M. Bouter, et al. Hyperglycaemia is associated with all-cause and cardiovascular mortality in the Hoorn population: the Hoorn Study.- Diabetologia, 42, 1999, No 8, 926-931.

7. Hu, D. Y., C. Y. Pan, J. M. Yu. The relationship between coronary artery disease and abnormal glucose regulation in China: the China Heart Survey.- Eur. Heart J., 27, 2006, No 21, 2573-2579.

8. Kempf, K., R. Füth, W. Dinh, L. Bansemir, T. Köhler, A. Bufe, et al. Screening for overt diabetes by oral glucose tolerance test: stratification by fasting blood glucose and patients' age improve practicability of guidelines in cardiological routine.- Int. J. Cardiol., 150, 2011, No 2, 201-205.

9. Lawes, C. M., V. Parag, D. A. Bennett, I. Suh, T. H. Lam, G. Whitlock, et al. Blood glucose and risk of cardiovascular disease in the Asia Pacific region.Diabetes Care, 27, 2004, No 12, 2836-2842.

10. Norhammar, A., A. Tenerz, G. Nilsson, A. Hamsten, S. Efendíc, L. Rydén, et al. Glucose metabolism in patients with acute myocardial infarction and no previous diagnosis of diabetes mellitus: a prospective study.- Lancet, 359, 2002, No 9324, 2140-2144. 
11. Qiao, Q., K. Pyörälä, M. Pyörälä, A. Nissinen, J. Lindström, R. Tilvis, et al. Two-hour glucose is a better risk predictor for incident coronary heart disease and cardiovascular mortality than fasting glucose.- Eur. Heart J., 23, 2002, No 16, 1267-1275.

12. Saely, C. H., H. Drexel, H. Sourij, S. Aczel, H. Jahnel, R. Zweiker, et al. Key role of postchallenge hyperglycemia for the presence and extent of coronary atherosclerosis: an angiographic study.Atherosclerosis, 199, 2008, No 2, 317-322.

13. Sourij, H., C. H. Saely, F. Schmid, R. Zweiker, T. Marte, T. C. Wascher, et al. Post-challenge hyperglycaemia is strongly associated with future macrovascular events and total mortality in angiographied coronary patients.- Eur. Heart J., 31, 2010, No 13, 1583-1590.
14. The DECODE study group, European Diabetes Epidemiology Group (Diabetes Epidemiology: Collaborative analysis Of Diagnostic criteria in Europe). Glucose tolerance and mortality: comparison of WHO and American Diabetes Association diagnostic criteria.- Lancet, 354, 1999, No 9179, 617-621.

15. Tominaga, M., H. Eguchi, H. Manaka, K. Igarashi, T. Kato, A. Sekikawa. Impaired glucose tolerance is a risk factor for cardiovascular disease, but not impaired fasting glucose: the Funagata Diabetes Study.- Diabetes Care, 22, 1999, No 6, 920-924.

16. Wascher, T. C., H. Sourij, M. Roth, P. Dittrich. Prevalence of pathological glucose metabolism in patients undergoing elective coronary angiography.- Atherosclerosis, 176, 2004, No 2, 419-421. 